\title{
Application of a dual-plane particle image velocimetry (dual-PIV) technique for the unsteadiness characterization of a shock wave turbulent boundary layer interaction
}

\author{
L J Souverein ${ }^{1,2}$, B W van Oudheusden ${ }^{2}$, F Scarano ${ }^{2}$ and P Dupont ${ }^{1}$ \\ ${ }^{1}$ Université d'Aix-Marseille-CNRS, UMR No 6595, 5 rue Enrico Fermi, 13453 Marseille Cedex 13, \\ France \\ ${ }^{2}$ Faculty of Aerospace Engineering, Delft University of Technology, Kluyverweg 1, 2629 HS Delft, \\ the Netherlands \\ E-mail: 1.j.souverein@tudelft.nl
}

Received 1 September 2008, in final form 22 December 2008

Published 21 May 2009

Online at stacks.iop.org/MST/20/074003

\begin{abstract}
The unsteady organization and temporal dynamics of the interaction between a planar shock wave impinging on a turbulent boundary layer at a free-stream Mach number of $M_{e}=1.69$ is investigated experimentally by means of dual-plane particle image velocimetry (dual-PIV). Two independent PIV systems were combined in a two-component mode to obtain instantaneous velocity fields separated by a prescribed small time delay. This enables us to obtain, in addition to mean and statistical flow properties, also instantaneously time-resolved data to characterize the temporal dynamics of the flow phenomenon in terms of time scales, temporal correlations and convective velocities. The characteristic time scales for the incoming boundary layer, the separation region and the reflected shock are determined by means of the temporal auto-correlation coefficient in the complete flow field for a range of time delays from $5 \mu$ s to $2000 \mu \mathrm{s}$. These auto-correlation fields are used to quantify the time scales in selected regions of the flow, with special interest for the vortex shedding and the low frequency reflected shock dynamics. This permits resolving the dominant time scales within the boundary layer and the interaction region.
\end{abstract}

Keywords: shock wave boundary layer interaction, unsteadiness, time scales, low frequency shock dynamics, PIV, dual-PIV

(Some figures in this article are in colour only in the electronic version)

\section{Nomenclature}

C second constant of the log-law

$C_{f} \quad$ friction coefficient

$\delta \quad$ boundary layer thickness (see $\left.\delta_{99}\right)$

$\delta_{99} \quad$ boundary layer thickness

$\delta t_{1} \quad$ pulse delay between image acquisitions

$\delta t_{2} \quad$ time separation between the PIV systems

$\delta^{*} \quad$ displacement thickness
FOV field of view

$f \quad$ focal length of the objective

$f_{\#} \quad f$-number, diffraction setting

$M_{e} \quad$ free-stream Mach number

$\operatorname{Re}_{\theta} \quad$ momentum-thickness-based Reynolds number

$R_{u_{1} u_{2}} \quad$ temporal auto correlation coefficient

$U \quad$ horizontal component of velocity

$U_{c} \quad$ convective velocity of structures 


$\begin{array}{ll}U_{e} & \text { free-stream velocity } \\ U_{v d}^{+} & \text {van Driest scaled velocity in wall units } \\ u^{\prime} & \text { horizontal turbulent fluctuation } \\ u_{\tau} & \text { friction velocity } \\ V & \text { vertical component of velocity } \\ v^{\prime} & \text { vertical turbulent fluctuation } \\ \text { PIV } & \text { particle image velocimetry } \\ p_{0} & \text { stagnation pressure } \\ \text { RMS } & \text { root mean square } \\ \sigma & \text { standard deviation } \\ T_{0} & \text { stagnation temperature } \\ \theta & \text { shock generator angle } \\ X & \text { horizontal coordinate } \\ Y & \text { wall normal coordinate } \\ y^{+} & \text {wall normal coordinate in wall units }\end{array}$

\section{Introduction}

The effect of a planar shock impinging on a turbulent boundary layer establishes one of the classic interaction phenomena in compressible viscous flow analysis. This particular form of interaction has also direct technological relevance to the performance of high-speed vehicles, affecting notably the efficiency of supersonic intakes. Furthermore, maximum mean and fluctuating pressure and thermal loads on a structure are most often found in the regions of shock wave boundary layer interaction (SWBLI) and are thus important factors in vehicle development. Flow control is seen as an important issue in future vehicle design (see Dolling (2001)) requiring a rigorous physical understanding of the mechanisms behind the phenomenon as well as improved simulation capabilities, both of which cannot be attained without fundamental experimental investigations and validation. The renewed attention for the feasibility of sustainable supersonic transport has revived the interest in SWBLI in the moderate supersonic regime. In this context, the European sixth framework program UFAST 'unsteady effects in shock wave induced separation' was recently initiated, in which the current shock reflection interaction is one of the flow cases of interest, with an emphasis on closely coupled numerical and experimental investigations (see Doerffer (2007)).

Conventional two-component PIV measurements have been reported that document both the statistical and instantaneous behaviour of shock wave boundary layer interactions (see for example Humble et al (2006)). Although these measurements provide a good idea of the overall flow organization, information is lacking on its temporal development, as characterized by quantities such as time scales, characteristic frequencies and the local acceleration. Given the multi-timescale nature of the phenomenon (see Dupont et al (2006)), knowledge of these quantities would profoundly increase the understanding of the flow, especially considering the correlation between events in different regions of the flow. In particular, for the flow under consideration, one can think of the relation between the motion of the reflected shock and upstream and downstream events (respectively the passage of turbulent structures through the interaction and the expansion and contraction of the separation bubble), as discussed by Dussauge et al (2006) and Ganapathisubramani et al (2007). Furthermore, the local acceleration field has an interest in its own right (see Perret et al (2006) and Christensen and Adrian (2002a, 2002b)), for example for the modelling of the structure of wall turbulence in the context of the improvement of subgrid-scale models for large-eddy simulations (LES). It may tentatively also enable compressible loads determination methods as discussed in Souverein et al (2007a), Van Oudheusden et al (2007) and Van Oudheusden and Souverein (2007) to be extended to include instantaneous loads and pressures (see Liu and Katz (2006)). However, due to the technical restrictions on both the double pulse repetition rate of the laser system and the acquisition rate of the cameras, the recording frequency is limited to the order of $10 \mathrm{~Hz}$ for conventional CCD-based PIV systems, and still to typically $1-10 \mathrm{kHz}$ for currently available CMOSbased high-speed PIV systems (at a significant reduction of image quality in terms of illumination power and spatial resolution). This is by far insufficient to obtain accurate time-resolved data for the high-speed flow case under investigation. The time scales in the shock wave turbulent boundary layer interaction typically span three orders of magnitude (see for example Dupont et al (2006)): $O(10 \mathrm{kHz})$ for the incoming boundary layer, $O(1 \mathrm{kHz})$ for the mixing layer developing inside the interaction and $O(100 \mathrm{~Hz})$ for the reflected shock motion. For example, for the flow under consideration the integral time scales in the incoming boundary layer are estimated at $24 \mu \mathrm{s}$, the associated frequency being $42 \mathrm{kHz}$ (see section 6).

To study the interaction with sufficient temporal resolution a dual-system PIV-approach was therefore applied, where two independent CCD-based PIV systems are combined to obtain instantaneously time-resolved whole field measurements, where the time delay between the acquisitions from both PIV systems could be set to arbitrarily small values, not limited by the repetition-rate restrictions of a single system. This way time correlated data could be obtained as well as acceleration data. The advantage of this approach with respect to the available high-speed PIV systems is in the first place that it benefits from the higher laser power and image resolution of the low rep-rate CCD systems. Secondly, it allows setting the delay time between the two PIV systems independent of the pulse separation of the individual systems, decoupling the temporal resolution of the time-resolved velocity fields from the time separation between the PIV image pairs, this being without consequence for the illumination power. A large range of time delays is therefore accessible, including extremely small delay times (below $100 \mu \mathrm{s}$ ). This gives the dual-PIV system the advantage of a quasi-infinite dynamic range, as opposed to the limited time interval between the high-repetition rate acquisitions. The smallest time separation employed in the current investigation was $5 \mu \mathrm{s}$, corresponding to an effective frequency of $200 \mathrm{kHz}$ (as opposed to acquisition rates in the order of $10 \mathrm{kHz}$ for available high-speed PIV systems). This allows temporally resolving the time scales within the boundary layer and within the interaction region.

Presented here are the results obtained with this dualPIV system. In the first place the system itself is discussed. 
Secondly, the quality and consistency of the dual-PIV measurements are considered, and the data are validated by means of a comparison to available boundary layer data, semiempirical relations and earlier SWBLI measurements. Thirdly, an overview is given of the obtained mean and fluctuation statistics, and the existence of instantaneous flow separation and convective vortices is evaluated. Finally, the temporal auto-correlation coefficients are obtained in the complete flow domain for different delay times. Characteristic time scales are derived from this for different regions in the flow, specifically for the reflected shock, the incoming boundary layer and the separation zone.

\section{Experimental arrangement}

\subsection{Flow facility}

The experiments were performed in the TST-27 transonic supersonic wind tunnel of the High-Speed Aerodynamics Laboratory at Delft University of Technology, with test section dimensions of $280 \mathrm{~mm}$ (width) $\times 270 \mathrm{~mm}$ (height). It is a blow-down facility that can operate at Mach numbers ranging from 0.5 to 0.85 and from 1.15 to 4.2 and a unit Reynolds number in the range of $30 \times 10^{6}$ to $130 \times 10^{6} \mathrm{~m}^{-1}$, with a run time of up to $300 \mathrm{~s}$. The Mach number is set by means of a continuously variable throat and flexible upper and lower nozzle walls. The stagnation pressure can be set independently with typical values ranging from 2.0 to 3.0 bar; the stagnation temperature is determined by the ambient temperature of the air storage vessel. The velocity transient inherent to blowdown operation has been determined to be approximately $5 \times$ $10^{-2} \mathrm{~m} \mathrm{~s}^{-2}$ corresponding to a total temperature transient of $6 \times 10^{-2} \mathrm{~K} \mathrm{~s}^{-1}$. The effect on the mean velocity is less than $1 \%$ of the free-stream velocity over a complete run duration of $60 \mathrm{~s}$.

During the current experimental investigation, the measurement conditions were a nominal free-stream Mach number of $M_{e}=1.69\left(U_{e}=448 \mathrm{~m} \mathrm{~s}^{-1}\right)$, a total temperature of $T_{0}=273 \mathrm{~K}$ and a total pressure of $p_{0}=2.3 \mathrm{bar}$ resulting in a free-stream unit Reynolds number of $36 \times 10^{6} \mathrm{~m}^{-1}$. The thickness of the tunnel wall boundary layer is $\delta_{99}=$ $17.2 \mathrm{~mm}$. The boundary layer was assessed to be in a fully developed turbulent condition with a Reynolds number based on the momentum thickness of approximately 50000. The incident shock wave for the SWBLI was generated by a fullspan wedge imposing a flow deflection of $\theta=6.0^{\circ}$ (see figure 1). The shock generator was mounted so as to position the interaction region in the centre of the field of view (FOV), as well as to postpone the interaction of the expansion fan emanating from the shoulder of the wedge with the recovering boundary layer (the estimated point of impact is $3 \delta$ behind the shock intersection point). A stable and reproducible flow was achieved over both sides of the wedge even at this relatively low Mach number, which is a condition where the wind tunnel is sensitive to choking.

The overall flow topology of the SWBLI under consideration can be observed from the time-mean representation given in figure 2 as determined using a

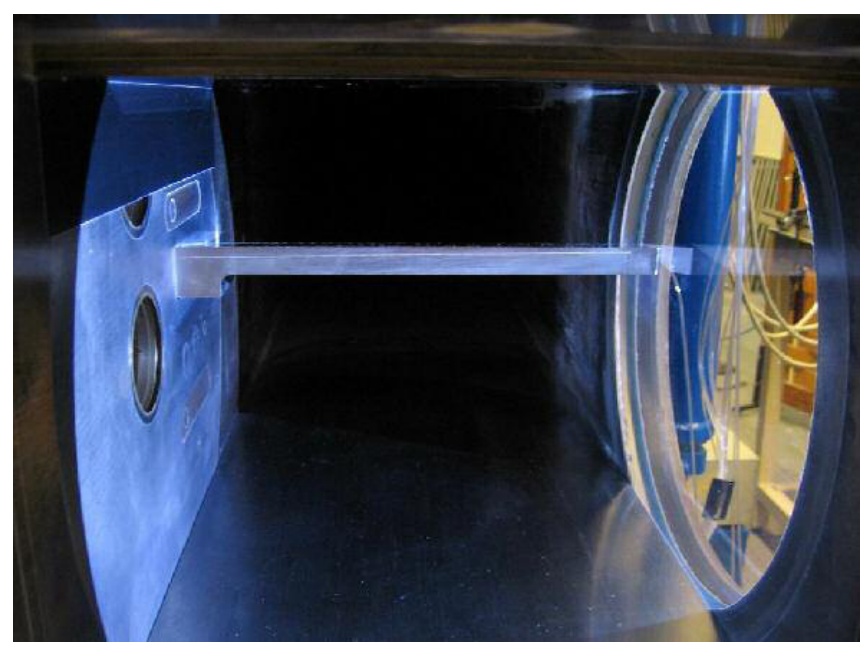

Figure 1. Experimental configuration: the test section with the sidewall mounted shock generator (span $280 \mathrm{~mm}$ ) as viewed from downstream. The viewing window is on the right.

standard two-component particle image velocimetry (2C PIV) technique with a wide view obtained by placing two cameras side by side. The homogeneous grey area delimiting the flow field indicates the tunnel wall and the edge of the optical access. The flow direction is from left to right and the contours depict constant values of the streamwise velocity component. The origin of the $(X, Y)$ coordinate system is taken at the extrapolated incident shock impingement point. It is noted that the small bumps in the contour levels at the wall at $X=-62 \mathrm{~mm}$ and $X=48 \mathrm{~mm}$ are measurement artefacts due to optical blooms on the wall. The irregularities in the contour lines at $X=-38 \mathrm{~mm}$ are due to the stitching procedure employed to construct the panoramic field of view. The incoming boundary layer can be discerned (the dashed horizontal line indicates the approximate boundary layer edge, which is about $17 \mathrm{~mm}$ from the wall), as well as the incident shock and the reflected shock. The interaction length, which is defined as the distance between the extrapolated intersections with the wall of the incident and reflected shock waves, is about $39 \mathrm{~mm}$ (or $2.2 \delta$ ). At the far right edge of the field of view the expansion fan emanating from the trailing edge of the shock generator is visible, which starts to interact with the boundary layer edge just over $2 \delta$ downstream of the extrapolated incident shock impingement point. The flow topology is typical for a reflected shock wave boundary layer interaction of moderate strength (see Smits and Dussauge (2006) and Smits (1997)), and is consistent with previous measurements performed in the same facility at $M_{e}=2.1$ (see Humble et al (2006)) and with observations in other experiments (see for example Dupont et al (2007)) and DNS and LES simulations (see Garnier and Sagaut (2002), Pirozzoli and Grasso (2006) and Touber and Sandham (2008)).

\subsection{Dual-PIV system arrangement}

The dual-PIV system was set up to acquire the locally timeresolved PIV data to obtain time correlated velocity field information. The rectangular contour in figure 2 indicates 


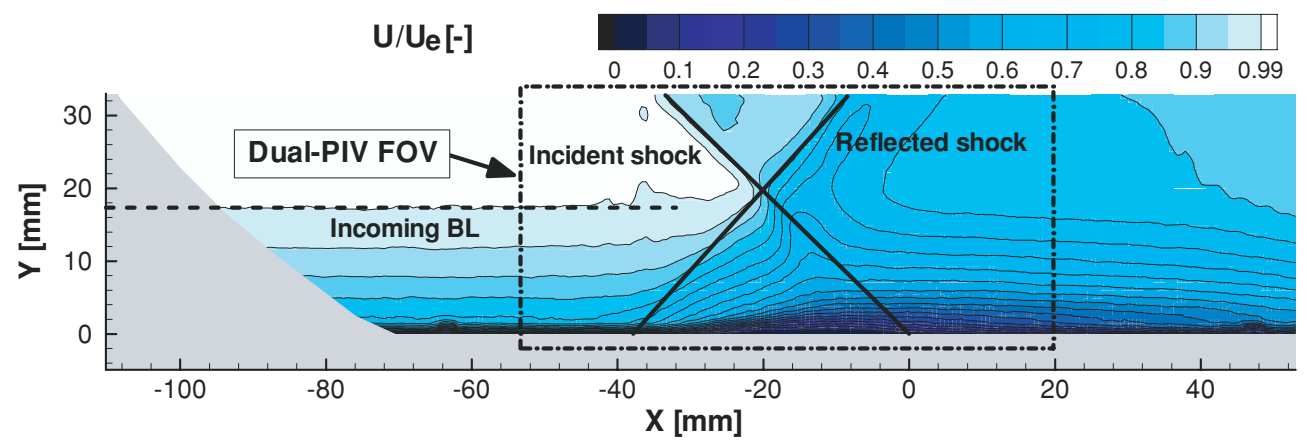

Figure 2. Panoramic field PIV measurement of the SWBLI under consideration.

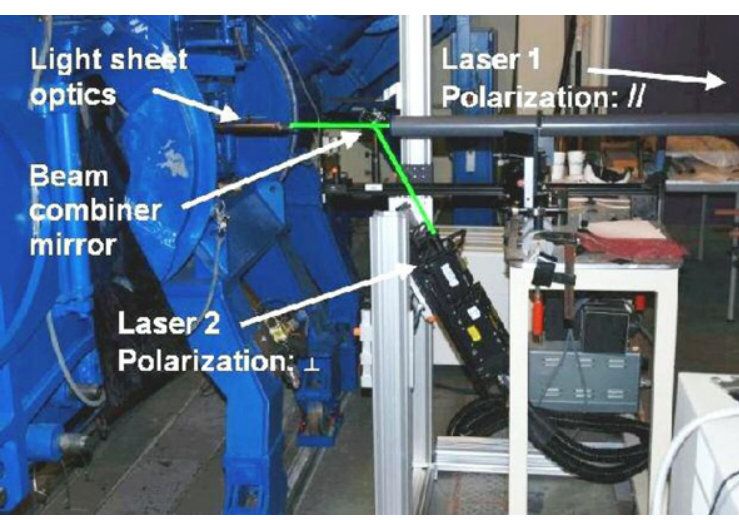

(a)

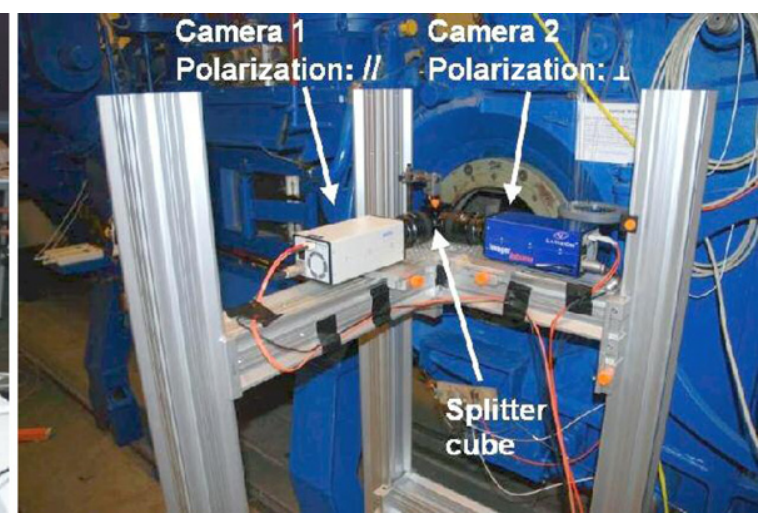

(b)

Figure 3. Experimental arrangement: (a) setup of the lasers with the beam combiner; $(b)$ setup of the cameras with the splitter cube.

the selected FOV for the dual-PIV measurements (note that the height extends to $Y=45 \mathrm{~mm}$, see figure 8 , which is beyond the range of figure 2). Illumination is provided from downstream of the test section while the observation is performed through a large window in the sidewall (see figure 1). The principle of dual-PIV depends on the mutually independent operation of two 2C PIV systems. Both systems are aligned to provide illumination in the same measurement plane while observing identical fields of view (see figure 3). The laser light of the two systems was optically distinguished by means of polarization and the beams of the two lasers were combined and aligned before entering into the light sheet optics. The overlap of the field of view of both cameras was guaranteed by means of a polarizing beam splitter cube, which also assured the independence of the two PIV systems by separating the images based on polarization. Both cameras were equipped with an additional polarizing filter with the aim of further improving the independence. Examples of equivalent systems can be found in Christensen and Adrian (2002a, 2002b), Liu and Katz (2006) and Kähler (2004), the latter using a stereoscopic implementation and two non-overlapping light sheets. Other dual plane PIV systems are described in Guibert and Lemoyne (2002) and Perret et al (2006), the latter being again a stereoscopic implementation. The applications range from the investigation of the spatio-temporal flow structure of turbulence, the determination of accelerations and the deduction of instantaneous pressure fields, notably in subsonic and stationary flow.

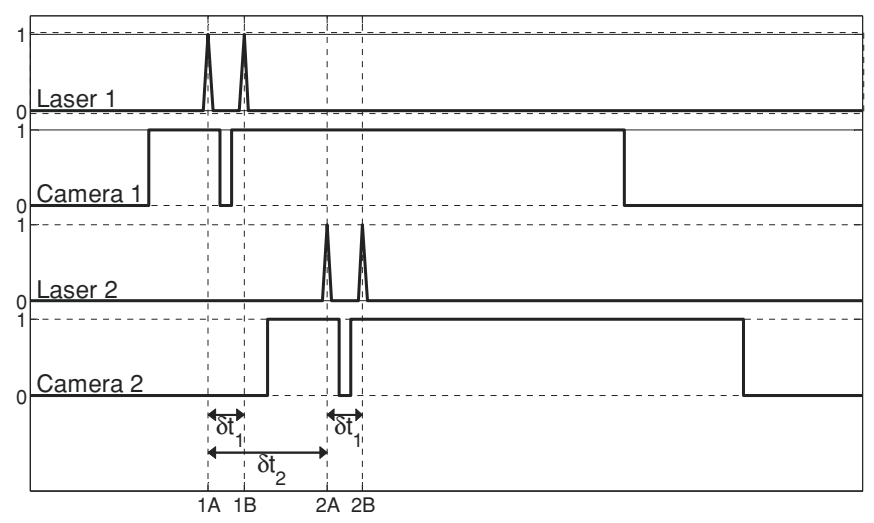

Figure 4. Dual-PIV timing diagram.

Care was taken in the current experiment that a total of four laser sheets generated by both lasers overlapped in space. Furthermore, special attention was dedicated to the temporal alignment of the laser pulses. The triggering was calibrated such that both lasers flashed simultaneously for a zero time delay between both systems $\left(\delta t_{2}=0 \mu \mathrm{s}\right)$ (see figure 4 for the timing diagram). This was done by means of a calibration run, determining the temporal off-set in the Qswitch trigger between both lasers through a correlation of the images corresponding to the respective laser pulses (i.e. image $1 \mathrm{~A}$ with image $2 \mathrm{~A}$, see figure 4).

The potential of the current setup becomes evident from the timing schematic in figure 4 , since for a given pulse delay 
$\delta t_{1}$ the time separation $\delta t_{2}$ can be set arbitrarily (and indeed even $\delta t_{1}$ for each PIV system individually as well, if desired). This allows obtaining time-correlated data at different time scales. In principle, measurements can be made with an unlimited temporal dynamic range, since $\delta t_{2}$ can be set to any value between zero and infinity. In the current experiment, a sweep of $\delta t_{2}$ was performed in the range of 5-2000 $\mu \mathrm{s}$ (corresponding to equivalent frequencies of $200 \mathrm{kHz}$ down to $0.5 \mathrm{kHz}$ ) with a reference measurement at $0 \mu$ s to check the consistency between the two PIV systems. A minimum of 200 acquisitions (400 image pairs) were made per time delay.

The illumination was provided by a Spectra-Physics Quanta Ray laser (400 mJ/pulse energy and $6 \mathrm{~ns}$ pulse duration) and a Quantel laser $(300 \mathrm{~mJ} /$ pulse energy and 9 ns pulse duration), installed as lasers 1 and 2 respectively. Both are double-pulsed Nd:YAG lasers with a wavelength of $532 \mathrm{~nm}$. The light sheet thickness was approximately $2 \mathrm{~mm}$. The flow was seeded with liquid DEHS (di(2ethylhexyl)sebacate) droplets, dispersed in the settling chamber of the wind tunnel using a PIVTEC seeding device. The estimated effective particle size is about $1 \mu \mathrm{m}$. The particle images were recorded at 12 bit with a resolution of $1376 \times 1040$ pixel using a PCO Sensicam QE (camera 1) and a LaVision Imager Intense QE (camera 2), both equipped with a Nikon $f=60 \mathrm{~mm}$ lens, diffracting with $f_{\#}=8$. Of the CDDs, only 992 pixels were used in the vertical direction given the aspect ratio of the interaction region of interest. The flow was imaged over a FOV of $76 \mathrm{~mm} \times 55 \mathrm{~mm}$ (approximately $4 \delta \times 3 \delta$ ) in streamwise and wall-normal directions, respectively, at a digital resolution of $55.1 \mu \mathrm{m} /$ pixel. The timing and data acquisition were performed by LaVision Davis 7.2 in combination with a PTU 9 timing unit.

Recordings were made at an acquisition rate of $5 \mathrm{~Hz}$. The pulse separation $\delta t_{1}$ was kept constant at $1.5 \mu \mathrm{s}$ for both laser systems, producing particle displacements of approximately $0.7 \mathrm{~mm}$ (corresponding to 12 pixels) in the free-stream flow. The image pairs were interrogated using the WIDIM algorithm (Scarano and Riethmuller 1999), employing correlation window deformation with an iterative multi-grid scheme, at $31 \times 31$ pixels window size $(1.7 \mathrm{~mm} \times$ $1.7 \mathrm{~mm}$ ) and an overlap factor of $75 \%$. This resulted in measurement grid resolution of $0.43 \mathrm{~mm}$ and $0.43 \mathrm{~mm}$ in the $X$ and $Y$ directions, respectively.

\section{Camera alignment}

Particular attention in setting up the measurement system was given to the alignment of the FOV of both cameras. The alignment of the cameras was assessed by means of the displacement field obtained for $\delta t_{2}=0 \mu \mathrm{s}$ and zero flow velocity (no airflow through the tunnel). The corresponding homogeneous deformation was obtained in terms of the dilatation, the rotation and the translation components as follows (see Gurtin (1981)):

$$
\mathbf{u}=(\mathbf{V Q}-\mathbf{I}) \mathbf{x}+\mathbf{c}
$$

where $\mathbf{u}$ is the displacement field, $\mathbf{V}$ is the left stretch tensor, $\mathbf{Q}$ is the rotation tensor, $\mathbf{I}$ is the unit tensor, $\mathbf{x}$ is the coordinate
Table 1. Homogeneous deformation results.

\begin{tabular}{llrl}
\hline \multirow{2}{*}{ Parameter } & \multirow{2}{*}{$\begin{array}{l}\text { Mean } \\
\text { field based }\end{array}$} & \multicolumn{2}{c}{ Instantaneous fields based } \\
\cline { 3 - 4 } & & Mean & RMS \\
\hline$\lambda(-)$ & 0.998 & 0.997 & $5 \times 10^{-5}$ \\
$\theta$ (degree) & 0.052 & 0.049 & 0.005 \\
$c_{x}$ (pixel) & 0.50 & 0.40 & 0.38 \\
$c_{y}$ (pixel) & -1.74 & -2.34 & 0.66 \\
\hline
\end{tabular}

vector and $\mathbf{c}$ is the translation vector. With $\mathbf{u}$ and $\mathbf{x}$ provided from measurement, the aim is to determine $\mathbf{V}, \mathbf{Q}$ and $\mathbf{c}$. The following system of equations results when assuming an isotropic deformation field:

$$
\left[\begin{array}{l}
u \\
v
\end{array}\right]=\left[\begin{array}{cc}
\lambda \cos \theta-1 & -\lambda \sin \theta \\
\lambda \sin \theta & \lambda \cos \theta-1
\end{array}\right]\left[\begin{array}{l}
x \\
y
\end{array}\right]+\left[\begin{array}{l}
c_{x} \\
c_{y}
\end{array}\right] .
$$

This is an over-determined system with the measured displacement field components $u$ and $v$ and coordinates $x$ and $y$ in terms of the scalar parameters $\lambda$ (dilatation) and $\theta$ (rotation), and the translation components $c_{x}$ and $c_{y}$. Since the origin is not prescribed by the problem, it was chosen at the centre of the field of view, which is considered to be the approximate physical centre of dilatation. The corresponding nonlinear least-squares optimization problem was solved by means of the Levenberg-Marquardt algorithm. Two approaches were followed: in the first place, the parameters of the deformation field were determined based on the mean displacement field (based on an ensemble of 75 realizations). Secondly, the same parameters and their mean and RMS values were also computed based on the instantaneous realizations. The deformation parameters thus obtained are listed in table 1.

Using the mean parameters based on the instantaneous velocity fields, the optimum interpolated deformation field was reconstituted. The computed displacement field based on the homogeneous deformation is found to be a good representation of the measured displacement field. A comparison of the reconstructed field to the measured field shows a discrepancy less than 1 pixel in absolute mean displacement.

Table 1 shows that the difference in magnification and rotation between the two FOVs is negligible: the difference in magnification is in the order of $0.1 \%$ and the rotation is in the order of $10^{-2}$ degrees. Furthermore, both the magnification factor and the angular alignment between both cameras are very stable, as may be concluded from the RMS values in table 1. The extremely small value of the RMS of the magnification factor may be considered a measure of the quality of the determination of the homogeneous displacement field parameters, since the magnification factor is a priori fixed by the camera objective lens, and the optical system is thought to be the least sensitive to vibrations in the out-of-plane direction. The horizontal and the vertical mismatch between the FOVs are in the order of 1 pixel with standard deviations that do not exceed 0.4 pixels and 0.7 pixels, respectively. In this respect it is reiterated that the location of the origin is not prescribed by the problem. Indeed, in Souverein et al (2007b), the origin was taken in the top-right corner yielding an absolute displacement of 2.85 pixels, as opposed to 1.81 pixels for the values in table 1 (mean field based). Depending on the choice 


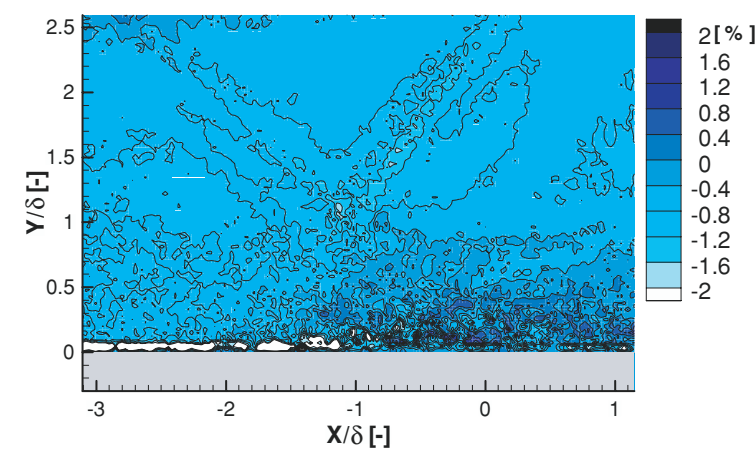

(a)

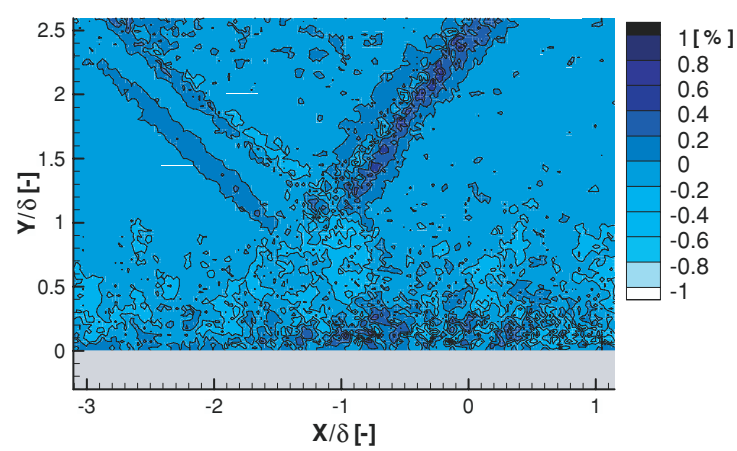

(b)

Figure 5. Difference between mean flow statistics obtained from each individual camera (values are given in percentages): $(a) \Delta U / U_{e}(\%)$; (b) $\Delta V / U_{e}(\%)$. Statistics based on 2000 acquisitions per camera. Spatial coordinates normalized by the boundary layer thickness $\delta_{99}$. Origin taken at the extrapolated incident shock foot.

of the origin within the FOV, the length of the vector $\mathbf{c}$ varies between the minimum value and the maximum value of the absolute misalignment ( 0.14 pixels to 4.3 pixels) with a mean value of 2.19 pixels. It may be concluded that the displacement mismatch is small as compared to the interrogation window size $(31 \times 31$ pixels $)$ and that the image of both cameras is dynamically stable to within a pixel accuracy.

Based on this analysis it is concluded that the physical alignment of the cameras was made to such a degree of accuracy that an image dewarp is not required.

\section{Data validation}

\subsection{Inter-camera data consistency}

Since the field of view is identical, the velocity statistics obtained from each PIV system should be identical to within the statistical convergence error. Therefore, a comparison has been made between the mean and fluctuation statistics obtained from each camera individually. The flow statistics obtained for the combined data from both cameras will be discussed in full detail in section 4 (see figures 8 and 9).

Figure 5 shows the difference in the mean velocity for both components $\left(\Delta U=U_{1}-U_{2}\right.$ and $\Delta V=V_{1}-V_{2}$, where subscript numbers indicate the respective cameras), expressed as a percentage of the free-stream velocity (refer to figure 8 for the combined mean fields). The difference between the two $U$-component fields is practically constant in most of the flow. It shows a bias error of the order of $0.7 \%$ (or $3 \mathrm{~m} \mathrm{~s}^{-1}$ ), which could be due to a small difference in camera alignment around the vertical axis (introducing a small viewing angle with respect to the flow plane) in combination with the slight difference in magnification mentioned in section 2. It is noted that the magnification factor discrepancy alone, due to its small value, is insufficient to explain the bias. The largest velocity differences occur in the regions with large gradients and large fluctuations (the reflected shock, the boundary layer and the interaction region), notably in the incoming boundary layer close to the wall where the velocity profiles are the fullest. The near-wall deviations are typically below $4 \%\left(18 \mathrm{~m} \mathrm{~s}^{-1}\right)$ and can be explained due to the combined action of the large velocity gradients close to the wall and the small misalignment mentioned in section 2; these errors at the wall do not occur within the interaction where the velocity profiles are much less full. It is noted that this region does not extend more than $2 \mathrm{~mm}$ away from the wall, which is approximately one iteration window size $(1.7 \mathrm{~mm})$. It can be seen that the $V$-component is identical to within $0.1-0.2 \%$ in most of the flow field. There is no observable bias error in the $V$-component in the free stream.

Figure 6 shows the difference between the measurements from both cameras of the fluctuation components $\left(\Delta \sigma_{u} / U_{e}\right.$, $\left.\Delta \sigma_{v} / U_{e}\right)$ and the Reynolds shear stress $\left(-\Delta\left\langle u^{\prime} v^{\prime}\right\rangle / U_{e}^{2}\right.$, where \langle\rangle represents the mean). As for the mean flow above, the data from the second camera are subtracted from the data obtained with the first camera. Note that the legend values are not presented in terms of percentages to allow a direct order of magnitude comparison with figure 9 . The velocity statistics from both cameras should again be identical, provided that convergence is reached.

The differences in the fluctuation component statistics are very small, at least one to two orders of magnitude smaller than the local absolute values of the statistics (see figure 9), and may be largely attributed to measurement noise and the fact that convergence is not yet achieved due to the limited ensemble size. This is underlined by the fact that the autocorrelation of the velocity fluctuations in the exterior flow, particularly for the $V$-component, is systematically larger for the first camera as compared to the second while the measured fluctuation values are close to zero in this part of the flow (see figure 9). This is consistent with an increase in the measurement uncertainty introduced by remnant traces of laser pulses from the second system in the second exposure of camera 1 (see figure 4). The fact that these fluctuations are measurement noise is confirmed by the fact that the Reynolds shear stress in the potential flow regions is zero, as is the difference between both cameras of this quantity. The additional fluctuations are hence not correlated. Furthermore, the hypothesis that a limited statistical convergence is at the source of the differences is strengthened by the fact that the largest deviations occur within regions with large fluctuations (boundary layer, mixing layer and vortex shedding) and display a granular pattern with a structure larger than the shift between the images. It is remarked that the fluctuation statistics and the Reynolds shear stress require a larger 


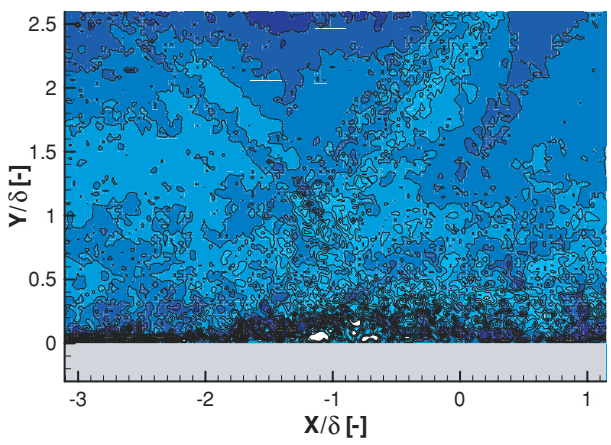

(a)
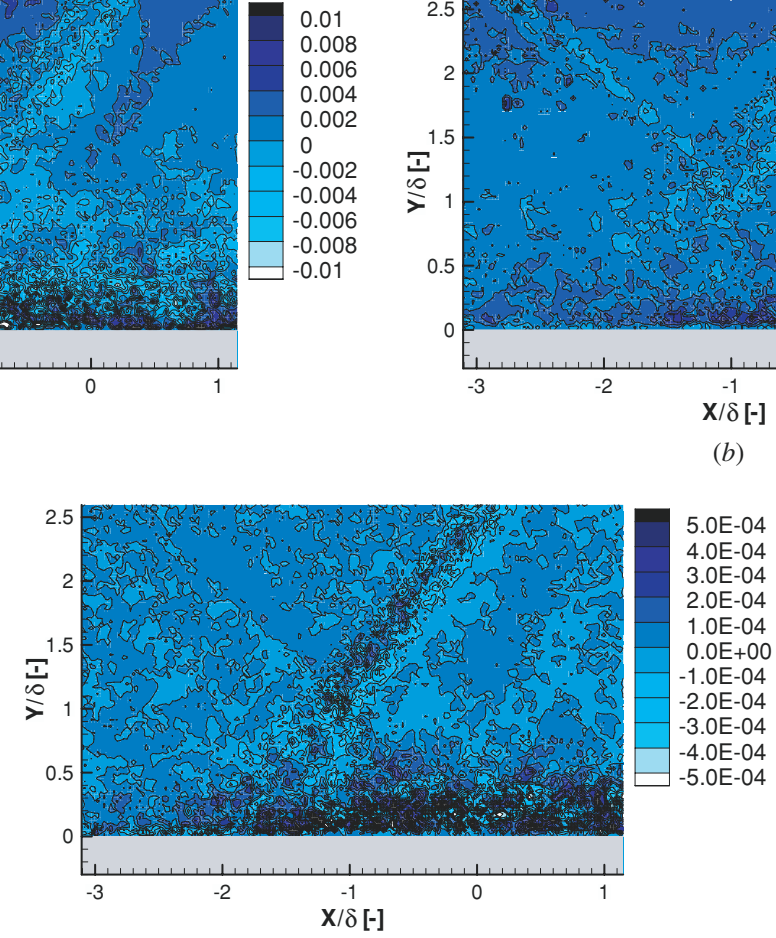

(c)

Figure 6. Difference between Reynolds stresses obtained from each individual camera: $(a) \Delta \sigma_{u} / U_{e} ;(b) \Delta \sigma_{v} / U_{e} ;(c)-\Delta\left\langle u^{\prime} v^{\prime}\right\rangle / U_{e}^{2}$. Statistics based on 2000 acquisitions per camera. Spatial coordinates normalized by the boundary layer thickness $\delta_{99}$. Origin taken at the extrapolated incident shock foot.

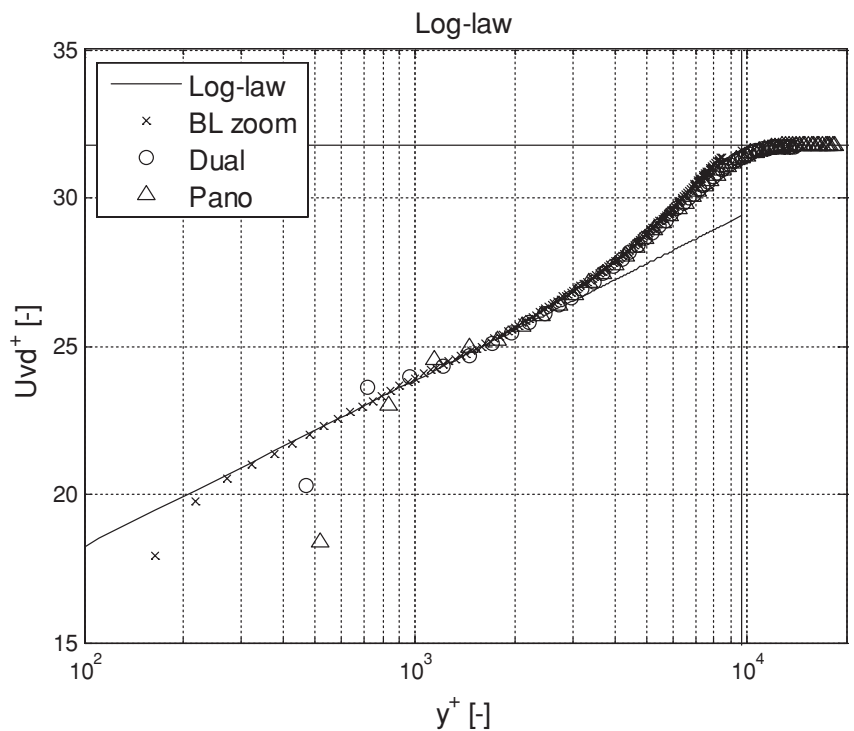

(a)

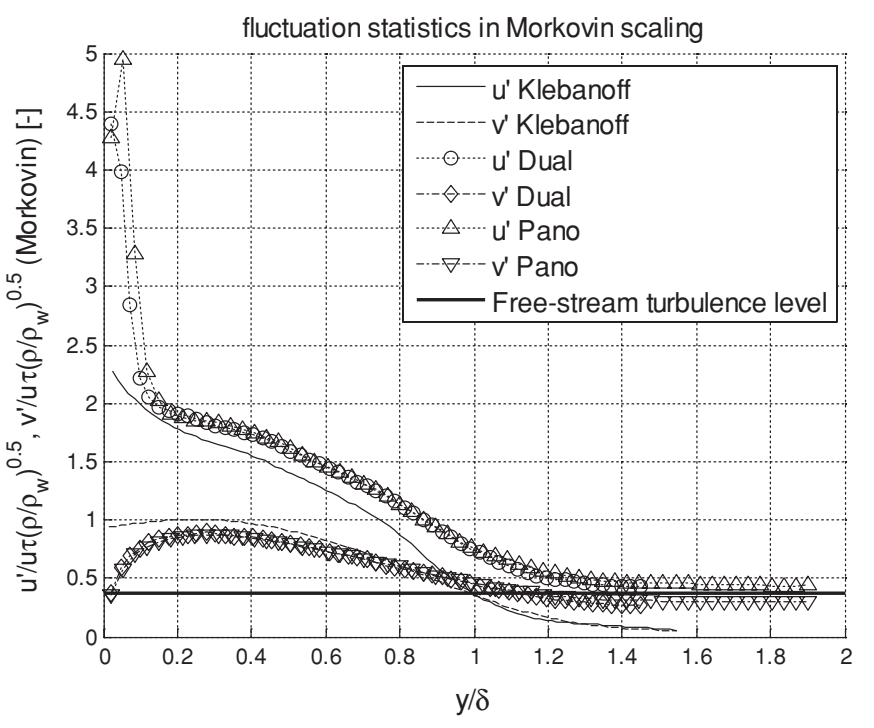

(b)

Figure 7. Boundary layer statistics: $(a)$ log-law of the wall, the solid horizontal and vertical lines indicate the free-stream velocity and boundary layer thickness, respectively; $(b) \sigma_{u}$ and $\sigma_{v}$ in Morkovin representation compared to Klebanoff reference data.

ensemble size to converge than the mean flow quantities. This is especially the case for the latter, which shows the most granular nature of all quantities, particularly in the interaction region and the recovering boundary layer where the velocity fluctuations are the largest. The only systematic deviation that can be attributed to the shift is found for $\sigma_{u}$ close to the wall in the incoming boundary layer at the same location where the maximum error in the mean $U$-component is found. This could be a result of a misalignment between the cameras since the autocorrelation of this component shows a very sharp rise close to the wall. It is hence particularly sensitive to small misalignments in this region, as is the mean $U$-velocity.

It is noted that the PIV measurement error on the velocity is approximately 0.1 pixel, or $0.8 \%$ of the free-stream velocity. Furthermore, the near-wall region (first iteration window) is a notoriously difficult region to obtain reliable PIV 
Table 2. Reference PIV measurement properties.

\begin{tabular}{lllll}
\hline Dataset & Short name & Ensemble size & Field of view $(-)$ & $\begin{array}{l}\text { Digital resolution } \\
(\mu \mathrm{m} / \text { pixel })\end{array}$ \\
\hline Dual-PIV & Dual & 4000 & $4 \delta \times 3 \delta$ & 55.1 \\
Panoramic FOV & Pano & 700 & $9 \delta \times 2 \delta$ & 70.1 \\
Boundary layer zoom & BL zoom & 250 & $0.7 \delta \times 0.9 \delta$ & 11.7 \\
\hline
\end{tabular}

Table 3. Determination of the friction coefficient $\left(\theta=1.39 \mathrm{~mm}, R_{\theta}=50000\right)$.

\begin{tabular}{lllllll}
\hline \multicolumn{2}{c}{ Experimental friction coefficient } & & \multicolumn{2}{c}{ Semi-empirical friction coefficient } \\
\cline { 1 - 2 } \cline { 5 - 6 } Case & $C_{f}(-)$ & $u_{\tau}\left(\mathrm{m} \mathrm{s}^{-1}\right)$ & & Reference & $C_{f}(-)$ & $u_{\tau}\left(\mathrm{m} \mathrm{s}^{-1}\right)$ \\
\hline Dual-PIV & $1.49 \times 10^{-3}$ & 15.07 & & Cousteix & $1.58 \times 10^{-3}$ & 15.50 \\
BL zoom & $1.48 \times 10^{-3}$ & 14.99 & & Fernholz & $1.46 \times 10^{-3}$ & 14.89 \\
Pano & $1.49 \times 10^{-3}$ & 15.06 & & & & \\
\hline
\end{tabular}

measurements, leading to increased measurement uncertainties and bias errors. Therefore it can be concluded that most of the deviations between the data from both cameras do not surpass the regular PIV measurement uncertainty level.

In conclusion, no significant tendency due to a misalignment affecting the topology and qualitative nature of the velocity statistics could be deduced from the above analysis. Small misalignments between the two cameras appear to affect mostly the flow within the first interrogation window next to the wall; the results obtained from each individual system are identical to within the statistical convergence in all other relevant parts of the flow. It is therefore concluded that the data are of sufficiently high quality.

\subsection{Physical compliance to reference data}

To further evaluate the measurement quality, a validation of the flow data is performed based on the incoming boundary layer profile and the statistical profiles within the interaction region. The data have also been compared to additional two-component PIV measurements of the same flow under identical measurement conditions, but at different spatial resolutions (see figure 7). The properties of the reference PIV measurements are summarized in table 2 (dual-PIV characteristics repeated for completeness). This validation is performed for the complete dual-PIV data ensemble, encompassing all acquisitions for both cameras combined in the statistical analysis (in total 4000 realizations) as an assessment of the quality of the dual-PIV measurements.

For the boundary layer profiles, the ensemble size has been augmented by taking statistics in the flow direction (over $0.7 \delta$ for the boundary layer zoom experiments, $0.6 \delta$ for the dual-PIV data and $1.5 \delta$ for the panoramic measurements) to increase the convergence and to attenuate measurement noise. It has been verified that this does not bias the results. As can be observed from figure 7 , none of the datasets resolves the log-law down to the viscous sub-layer. This is a consequence of the limited PIV resolution in combination with the high Reynolds number: $y^{+}=30$ corresponds to $53 \mu \mathrm{m}$, which equals 1 pixel at the dual-PIV magnification. The first reliable velocity measurement in the current experiment is at $y / \delta=$ 0.1 , or $y^{+}=1000$ (corresponding to one interrogation window size). The velocity fluctuations in Morkovin scaling (Smits and Dussauge 2006) are in good agreement with the Klebanoff (1953) reference data (note that the horizontal line in figure 7 indicates a $1 \%$ turbulence intensity in the free stream). The $U$-component fluctuations are resolved with good confidence down to $y / \delta=0.1$. The Reynolds stress was found to show reasonable agreement with the Klebanoff profile down to $y / \delta=0.3$ (see Souverein et al (2008)). Below this height the stress is underestimated, which may imply that the Reynolds shear stress measurements near the wall suffer from a systematic measurement error. The Reynolds stresses seem to be the most difficult quantities to measure with PIV in high-speed flows. Special attention is drawn to the fact that the dual-PIV data compare very well with the boundary layer zoom data acquired at a five times higher spatial resolution (see table 2), confirming that the same flow physics are captured. The dual-PIV measurements are consistent with all earlier experiments.

A consistency check has been performed of the friction coefficient and friction velocity obtained from a log-law fit (Smits and Dussauge 2006) with respect to results from semi-empirical relations (Cousteix 1989, Fernholz 1971) (see table 3). Good agreement was found both with the earlier measurements and with the semi-empirical correlations. The log-law fit was made without a priori fixing the second constant $C$. The best fit was obtained for $C=7$ and $u_{\tau}=$ $15 \mathrm{~m} \mathrm{~s}^{-1}$.

The obtained mean and fluctuating velocity profiles, as well as the Reynolds shear stress profiles, at different streamwise locations throughout the interaction have been compared to measurements at larger and smaller magnification factors. Details can be found in Souverein et al (2008). It was observed that very good agreement is obtained for all datasets, confirming the reproducibility of the flow conditions for the shock wave boundary layer interaction under consideration.

In summary, the dual-PIV boundary layer measurements are found to be consistent with the earlier measurements and the obtained friction coefficient and friction velocity are in good agreement with the results obtained with the semiempirical relations. Furthermore, apart from the measurement 


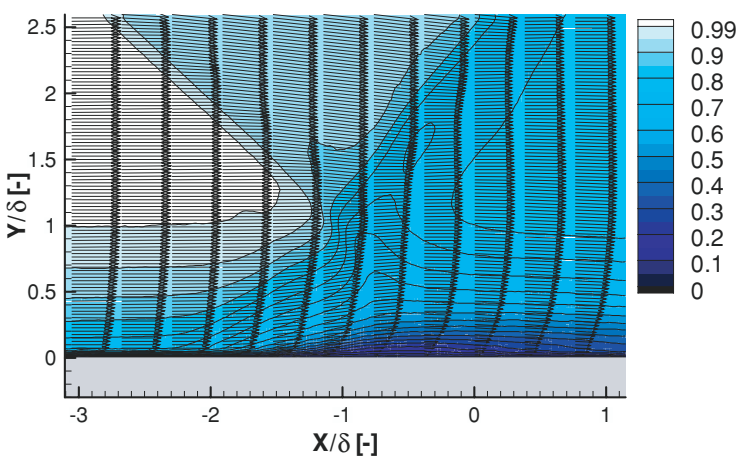

(a)

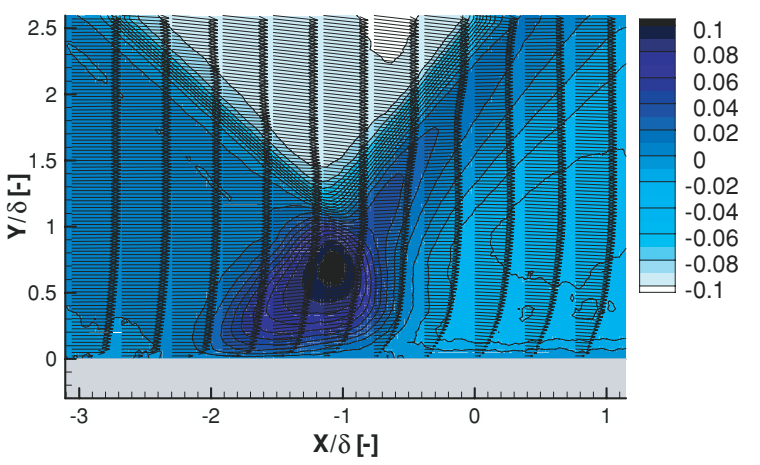

(b)

Figure 8. Non-dimensional mean velocity fields: (a) $U$-component $U / U_{e}(b)$ and $V$-component $V / U_{e}$. Statistics based on 4000 acquisitions. Spatial coordinates normalized by the boundary layer thickness $\delta_{99}$. Origin taken at the extrapolated incident shock foot.

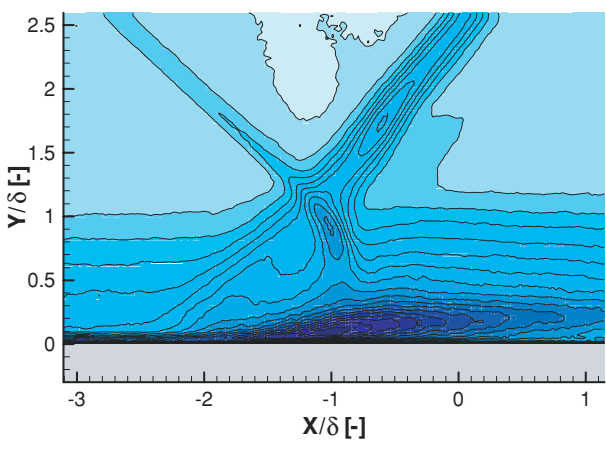

(a)
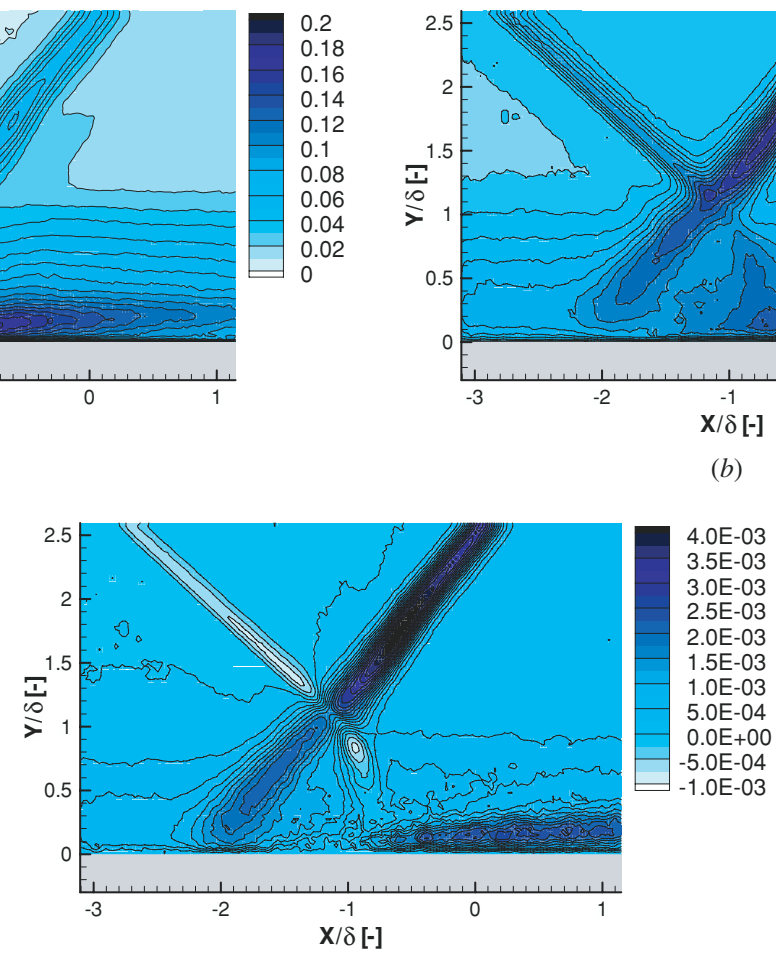

(c)

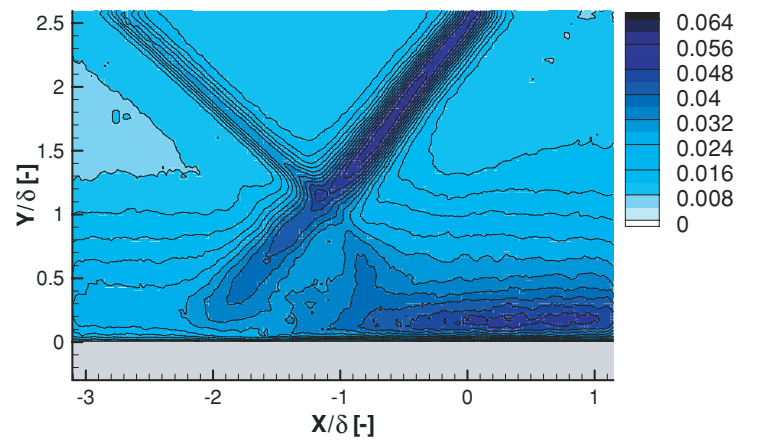

(b)

Figure 9. Non-dimensional Reynolds stresses: (a) $\sigma_{u} / U_{e} ;(b) \sigma_{v} / U_{e} ;(c)-\left\langle u^{\prime} v^{\prime}\right\rangle / U_{e}^{2}$. Statistics over 4000 acquisitions. Spatial coordinates normalized by the boundary layer thickness $\delta_{99}$. Origin taken at the extrapolated incident shock foot.

points close to the wall, no dependence of the flow quantities on the spatial resolution was observed. The flow conditions were found to be in accordance with other datasets. It can thus be concluded that the dual-PIV dataset is of consistent quality and the spatial resolution is sufficient to capture the relevant flow physics. Further, the incoming boundary layer under consideration is a canonical turbulent boundary layer and the flow conditions are repeatable.

\section{Mean and fluctuation statistics}

Figures 8 and 9 show the mean velocity field and the fluctuating velocity statistics respectively, providing a global understanding of the flow topology under consideration. The thickness of the undisturbed boundary layer is $\delta_{99}=17.2 \mathrm{~mm}$.
The interaction length based on the distance between the extrapolated point of impact of the incident shock and the reflected shock foot is approximately $2 \delta$. The figures show that the incoming boundary layer remains undisturbed within at least the first $10 \mathrm{~mm}(0.6 \delta)$ of the FOV. The incident shock wave was observed to be stationary and the local velocity fluctuations are mainly attributed to PIV measurement uncertainties in the direct vicinity of the shock. The reflected shock shows strong velocity fluctuations as a consequence of pronounced variations in the shock positions. The extent of the reflected shock excursions is estimated, based on the vertical velocity component fluctuations, as approximately $\pm 5 \mathrm{~mm}$ $( \pm \delta / 3)$. Both shocks are further smoothed in the mean velocity fields due to a combination of particle inertia, optical refraction effects (see Elsinga et al (2005)) and the limited PIV spatial 
and temporal resolution (due to central differencing over the time separation $\delta t_{1}$ between the image pairs). The increase in $V$-component fluctuations observed upstream of the incident shock is also due to an optical refraction effect, probably due to density gradients within the optical path caused by the interaction of the shock with the boundary layer on the tunnel side window (see figure 1). The particle images in this region are blurred with no physical change in the flow velocity.

Considering the external flow outside the boundary layer, it can be seen that the flow is initially parallel to the wall and consecutively decelerated by the shock system. The first (impinging) shock causes a downward deflection resulting in a negative vertical velocity of approximately $-45 \mathrm{~m} \mathrm{~s}^{-1}$ or about $10 \%$ of the free-stream velocity. The second (reflected) shock causes an upward deflection, which is directly followed by an acceleration caused by the expansion fan (which is the actual physical reflection of the incident shock wave). The expansion fan is practically attached to the reflected shock and deflects the flow to a small negative vertical velocity component of approximately $-13 \mathrm{~m} \mathrm{~s}^{-1}$ or $3 \%$ of the freestream velocity. Considering the boundary layer, the strong adverse pressure gradient imposed by the shocks causes it to thicken dramatically, driving it towards separation. However, even though a large region of slow moving fluid exists close to the wall and flow reversal occurs instantaneously, the flow was not observed to be separated on the mean. An increase in the velocity fluctuations and the Reynolds shear stress throughout the interaction region can be observed. The large increase in the $U$-component fluctuations directly behind the reflected shock foot is associated with the pulsation of an instantaneous separation bubble. The large $U$-component fluctuations and the increase in $V$-component fluctuations and Reynolds shear stress are the characteristics of vortical structures developing within a mixing layer. Anisotropy can be observed between the streamwise and lateral fluctuation components since the maximum value attained by $\sigma_{u}$ is about four times higher than the value attained by $\sigma_{v}$. In contrast, the $U$-fluctuation recovers much faster than the $V$-fluctuation, which is seen to persist until the end of the observation domain. The existence of an instantaneous separation bubble and the presence of a mixing layer, as indicated by these observations, will be investigated in the following section.

\section{Characterization of the unsteady flow behaviour}

Although from the flow properties given in the previous section the flow appears to be non-separated on the mean, individual flow realizations reveal that significant reversed flow can occur instantaneously, suggesting that the present interaction is one of incipient separations. As further evidence to the separation dynamics, figure 10 shows the rate of the instantaneous occurrence of flow separation for each point in the flow domain, computed as the ratio of the number of realizations with locally reversed flow over the total number of realizations (the sonic line has been obtained using the modified Crocco-Busemann relation assuming adiabatic wall conditions, see White (1991)). It is clear that flow reversal occurs in up to $40 \%$ of the flow fields. These observations

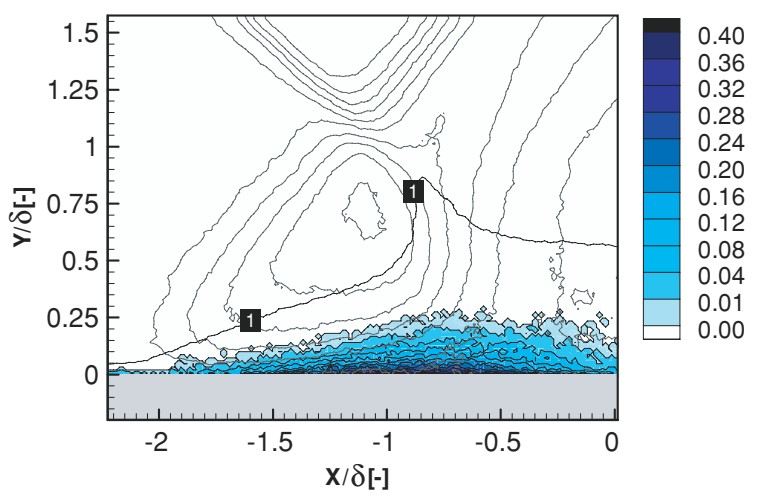

Figure 10. Instantaneous separation rate with for reference the contour lines of the vertical velocity component and the location of the sonic line (labelled with ' 1 ').

give evidence of the significant temporal dynamics governing the interaction even for this mild interaction strength.

Although the range of dynamic excursions in the instantaneous flow organization may already be appreciated from an ensemble of uncorrelated flow realizations, obtained with a low repetition rate measurement system (see also Humble et al (2006)), the dual-PIV system approach now allows us, in addition, to extract information on the temporal development of flow features in the interaction, permitting the determination of properties such as local acceleration, convective velocities and time correlation.

As a first illustration of the capabilities of the dual-PIV approach, some individual flow realizations are considered in further detail. Subsequently, in the next section a further quantification is made of the time scales of the different aspects in the flow dynamics (incoming boundary layer, reflected shock motion, separation region pulsation, shedding of vortical structures).

An example of the instantaneous occurrence of separation and the convection of vortical structures in the separated shear layer is shown in figure 11(a), together with its time-resolved counterpart in figure $11(b)$, captured with a time delay of $10 \mu \mathrm{s}$. The flow can be seen to display reversal, as indicated by the inflection point in the velocity profiles and the occurrence of negative velocity values (saturation of the zero velocity contour level in black). The maximum absolute value of the reversed flow velocity is $27.0 \mathrm{~m} \mathrm{~s}^{-1}\left(6.0 \%\right.$ of $\left.U_{e}\right)$ for the first realization and $34.3 \mathrm{~m} \mathrm{~s}^{-1}\left(7.7 \%\right.$ of $\left.U_{e}\right)$ for the second realization. The flow fields of figures $11(a)$ and $(b)$ are selected for a closer investigation to identify the existence of convected vortical structures. To this purpose a zoom is made of the velocity fields, as indicated by the rectangular frame. The two consecutive velocity fields are temporally separated by a time $\delta t_{2}=10 \mu$ s and a convective velocity of $290 \mathrm{~m} \mathrm{~s}^{-1}$ is subtracted to optimally visualize the vortices (see figures $11(c),(d)$ ). Two neighbouring vortex cores were identified in the first velocity field (indicated by the two solid circles, figure $11(c)$ ). They can be seen to convect in the streamwise direction by slightly under $3 \mathrm{~mm}$ within the time delay (original location indicated by the dashed circles, new location by the solid circle, figure $11(d)$ ), which is consistent with the estimated value of the convective velocity. Within the line of zero streamwise velocity another 


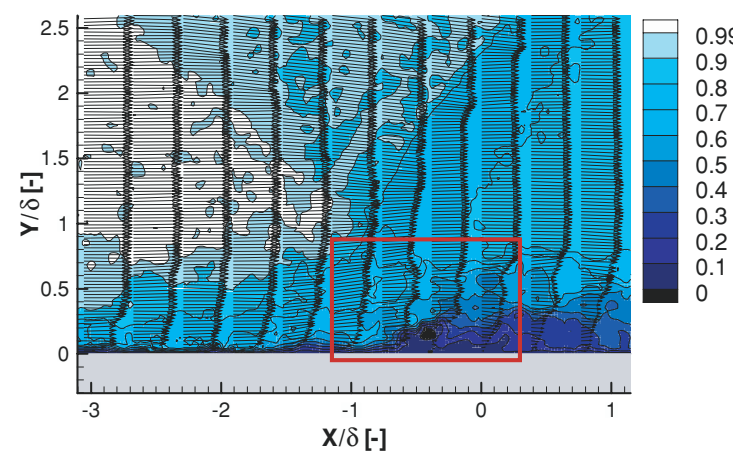

(a)

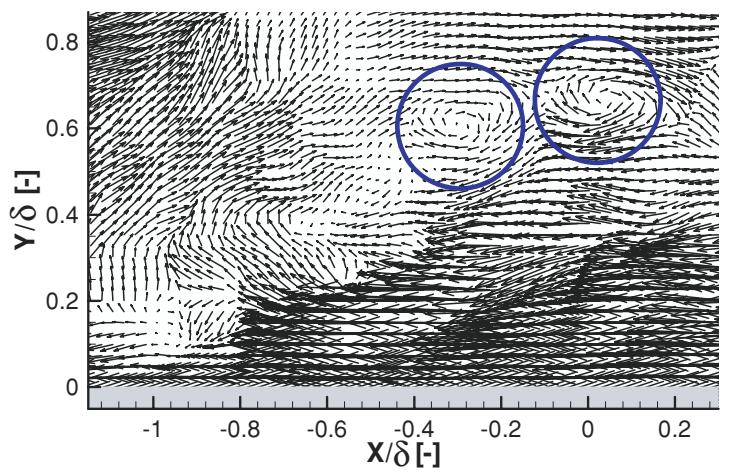

(c)

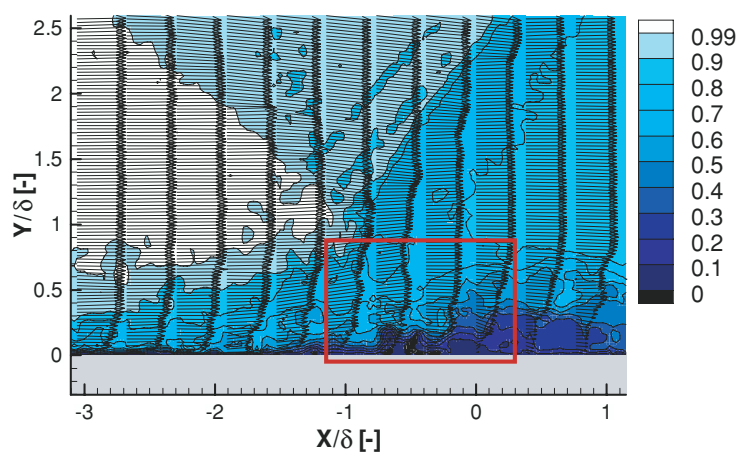

(b)

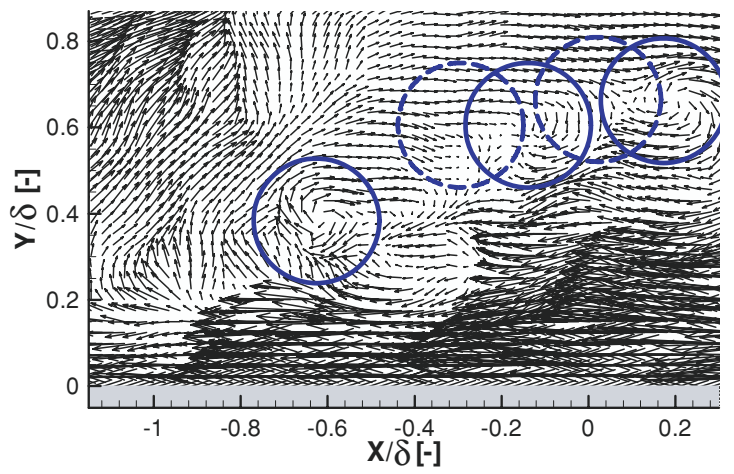

(d)

Figure 11. Instantaneous velocity fields: horizontal velocity component contours with velocity vectors, first realization $(a)$ and second realization $(b)$ separated by $\delta t_{2}=10 \mu \mathrm{s}$; zoom of the interaction region of the same acquisitions showing velocity vectors with a convective velocity of $290 \mathrm{~m} \mathrm{~s}^{-1}$ subtracted, first realization $(c)$ and second realization $(d)$.

vortex core was identified in the second field that could not be matched to a vortex in the first field (solid circle, figure $11(d)$ ). This confirms that vortical structures exist within the interaction region and that their downstream convection with a convective velocity of several hundreds of meters per second can be resolved with dual-PIV.

\section{Determination of time scales}

Since measurements were performed for a range of time separations from $\delta t_{2}=0 \mu \mathrm{s}$ to $2000 \mu \mathrm{s}$, this allows time correlation data for the complete flow field to be obtained as a function of the time delay. This can then be exploited to determine the characteristic time scale at each position in the flow. Specific regions of interest are the incoming boundary layer, the recirculation region where vortex production and shedding occurs, the reflected shock and the recovering boundary layer. Figures 12 and 13 show the time correlation coefficient for the $U$-component of velocity, for small and large time delays respectively. The time correlation coefficient is defined by equation (3), where $u_{i}^{\prime}$ is the fluctuation component and $\sigma$ represents the standard deviation. Furthermore, the indices 1 and 2 stand for the first and second measurements at a single point in the flow field, separated by $\delta t_{2}$ :

$$
R_{u_{1} u_{2}}=\frac{\overline{u_{1}^{\prime} u_{2}^{\prime}}}{\sigma_{u_{1}} \sigma_{u_{2}}} .
$$

This correlation coefficient may be interpreted as a measure for assessing the duration of coherence of a flow phenomenon at a fixed spatial coordinate. One can think of observing the passage of a vortical structure in the incoming boundary layer from a stationary point in space. For very small $\delta t_{2}$ (i.e. much smaller than the passing time of the vortex) the flow structure will hardly have moved and a very high correlation coefficient is obtained. For increasingly larger $\delta t_{2}$ the vortex will displace over a larger distance and the correlation coefficient will decrease accordingly, until the vortex has moved out of sight and hence the correlation vanishes. A similar reasoning can be applied to other flow features as well. Low frequency phenomena or long wavelength flow structures will lead to higher values of the correlation coefficient at large $\delta t_{2}$.

An effect that has to be taken into account in the computation of the correlation coefficient is the slow temporal drift in the free-stream velocity inherent to blow-down facilities as a consequence of the transient total temperature condition in the supply vessel (see section 1). The effect on the mean velocity is inferior to the free-stream turbulence and the measurement uncertainty. Nonetheless, a velocity trend does constitute a coherent very low frequency flow motion. In addition, combining multiple runs to compute the correlation statistics also introduces a low frequency artefact which is an indirect consequence of the velocity trend. Both effects could result in a residual non-zero value of the correlation coefficient at large $\delta t_{2}$. To remedy this, the velocity data have been corrected for any linear trends. The time correlation statistics have consecutively been computed for the correctly zero mean centred velocity fluctuations.

In the first place, it is observed that for $\delta t_{2}=0 \mu \mathrm{s}$, in which case both measurements should be identical and thus 

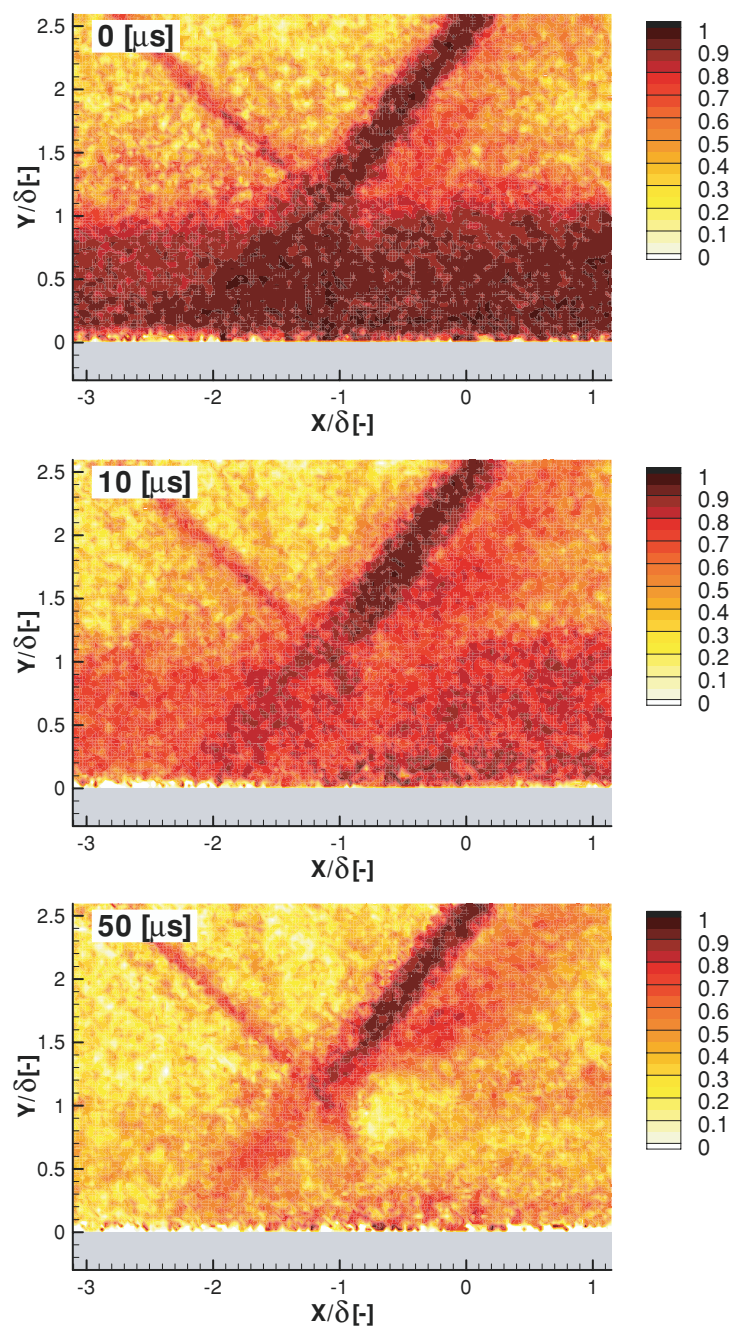
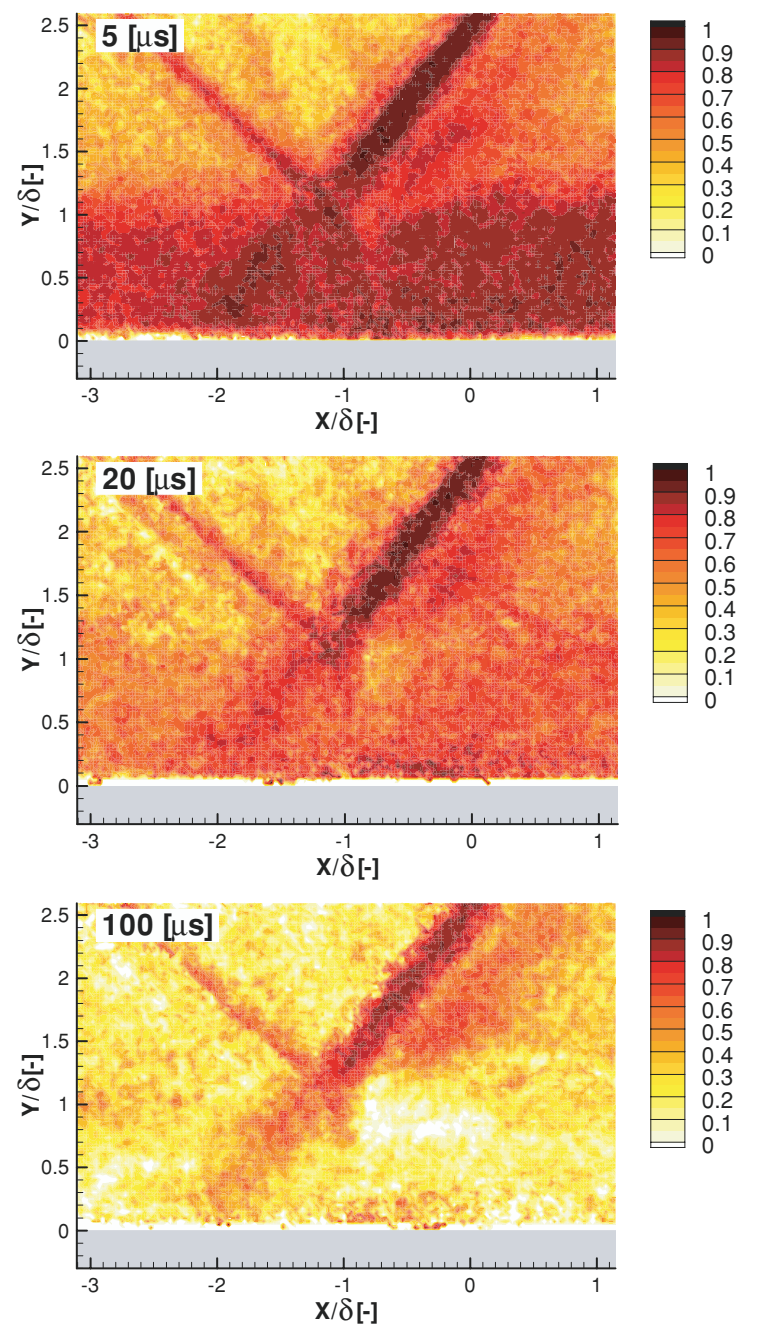

Figure 12. The temporal auto-correlation coefficient for small time delays ( $\delta t_{2}=0$ to $100 \mu \mathrm{s}$ ).

have a correlation coefficient of unity, not all of the flow is fully correlated. Especially in the external flow, where the fluctuations are small, the correlation is medium to low. This may be attributed to measurement noise and may indeed be interpreted as a measure of the accuracy of the PIV technique. As a second remark, it may be noticed that the incident shock wave appears in the correlation results for small $\delta t_{2}$. Since the incident shock was verified to be steady and since it appears for the zero-time-delay case as well, this feature may also be attributed to limitations inherent to the PIV measurements technique close to the shock, as was observed in the case of the Reynolds stresses.

A qualitative evaluation of the correlation coefficient in figures 12 and 13 shows that for small $\delta t_{2}$ below $10 \mu \mathrm{s}$ all regions of interest (the incoming boundary layer, the interaction zone and the reflected shock in combination with the expansion fan) remain highly correlated with values close to unity. Evidently, on the scale of the measurement resolution, no flow regions display time scales that are substantially below $5 \mu \mathrm{s}$, justifying the choice of this time delay as the smallest value in the investigation. The incoming boundary layer is the first region to decorrelate, starting from $\delta t_{2}=10 \mu \mathrm{s}$. The boundary layer is largely decorrelated at $\delta t_{2}=50 \mu \mathrm{s}$. At this time delay the interaction zone with the mixing layer and the subsequent vortex shedding and recovering boundary layer are still correlated. Somewhere between $\delta t_{2}=100 \mu \mathrm{s}$ and $500 \mu \mathrm{s}$, the mixing layer and vortex shedding regions also become decorrelated. The reflected shock shows high values of the correlation coefficient throughout most of the range of time delays considered so far. Only for the very large time delays does it start to decorrelate. The correlation coefficient of the reflected shock has practically vanished at $\delta t_{2}=2000 \mu \mathrm{s}$. This confirms the existence of different time scales within the flow domain, and in particular it evidences the low-frequency behaviour of the reflected shock.

This qualitative evaluation of the time scales can be quantified further by plotting the local time correlation coefficient against the time delay for different locations in the flow (see figure 14). The selected regions of interest are shown on the top of this figure (superimposed on the autocorrelation coefficient at $\delta t_{2}=200 \mu \mathrm{s}$ and contours of the Reynolds shear stress for reference; the dashed horizontal line indicates the approximate edge of the undisturbed boundary layer). Statistics have been computed over multiple points within each region of the flow to aid the statistical convergence (note that only 200 realizations are available per time delay). 

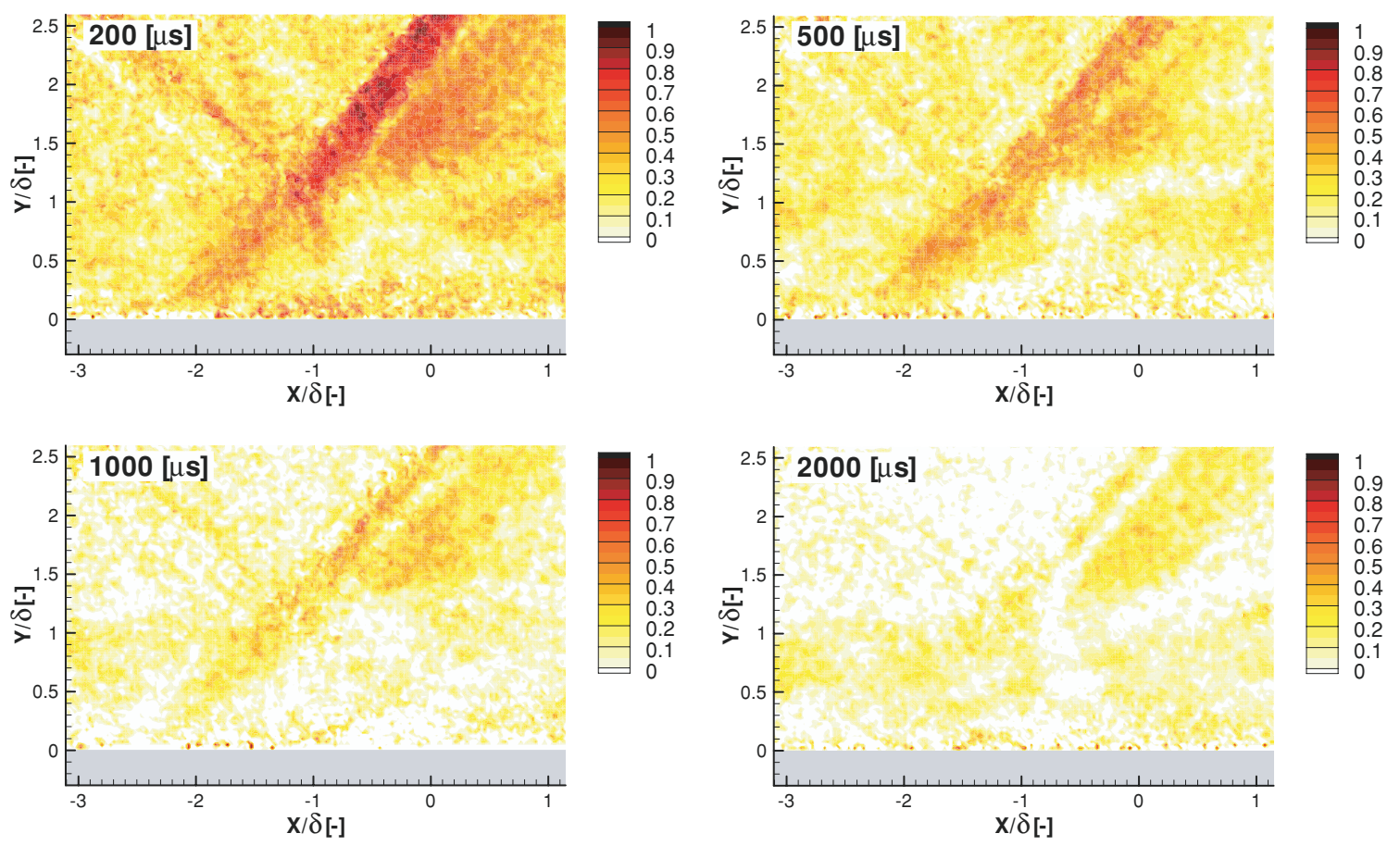

Figure 13. The time correlation coefficient for large time delays ( $\delta t_{2}=200 \mu \mathrm{s}$ to $2000 \mu \mathrm{s}$ ).

The fluctuations in each point are centred locally around a zero mean value by means of the aforementioned detrend routine. The correlation statistics are computed consecutively, treating the combination of points as a single data ensemble while taking into account the proper weighting for rejected vectors. It has been verified that no significant dependence of the results exists on the selection of each region within the local flow domain under consideration. For reference, the auto-correlation coefficient in the free stream is also shown. The results have been plotted in both linear and semi-log representations, the latter to better visualize the different orders of magnitude of the time scales.

The different orders of magnitude are clearly apparent from figure 14. The auto-correlation coefficients of the reflected shock have not yet vanished for the large time delays, while the values of the other regions have already converged asymptotically to zero at approximately $\delta t_{2}=1000 \mu \mathrm{s}$. Taking the crossing of the $1 / \mathrm{e}$-level of the auto-correlation coefficient as representative for the time scales, it is evident that the smallest time scales are found in the incoming boundary layer, followed by the recovering boundary layer, the vortex shedding, the reflected shock foot and the reflected shock. Looking at the incoming boundary layer, a characteristic time is obtained of $40 \mu$ s and hence an equivalent frequency of $25 \mathrm{kHz}$. Considering an integral length scale of $\Lambda / \delta=0.5$ (see Dussauge and Smits (1995)) and a convective velocity of $0.8 U_{e}$ yields an integral time scale of $24 \mu$ s and hence a frequency of $42 \mathrm{kHz}$. The boundary layer time scales hence appear to be overestimated. It is unknown if this overestimated value is physical or whether it is a measurement artefact. As for the vortex shedding, a characteristic time scale of $80 \mu \mathrm{s}$ is obtained, corresponding to $12.5 \mathrm{kHz}$. For the reflected shock, a time scale of $800 \mu \mathrm{s}$, or $1250 \mathrm{~Hz}$, is obtained. Both the measured equivalent frequency of the vortex shedding and that of the reflected shock are of approximately the same order of magnitude as the values reported in the literature (see Dupont et al (2006)).

Considering figure 14 in more detail, two interesting observations can be made. In the first place, the reflected shock foot, the vortex shedding and the recovering boundary layer regions all show the same behaviour for small time delays, and further follow more loosely the trend of the incoming boundary layer. For larger time delays, the downstream boundary layer seems to tend towards the incoming boundary layer, diverging from the vortex shedding trend. This is indeed indicative of the recovery process of the boundary layer. For larger time delays, the incoming boundary layer, the vortex shedding and the recovering boundary layer curves are more difficult to interpret since they become of the same order of magnitude as the free-stream value, i.e. essentially losing correlation. It is noted however that the auto-correlation value itself in the free stream is very small (one to two orders of magnitude smaller in comparison to the other regions).

A second important observation is that the reflected shock foot auto-correlation coefficient displays behaviour intermediate to the incoming boundary layer and the reflected shock in the outer flow. At small time delays, the reflected shock foot responds rather similar to the incoming boundary layer while at larger time delays the reflected shock foot seems to behave like the reflected shock in the external flow. Hence, even though the signal is influenced by the passage of turbulent structures present in the incoming boundary layer, there is also a strong low-frequency component. This indicates that the reflected shock foot moves in harmony with the low frequency motion of shock in the outer flow. Similar behaviour has indeed been observed in experiments and LES simulations of 


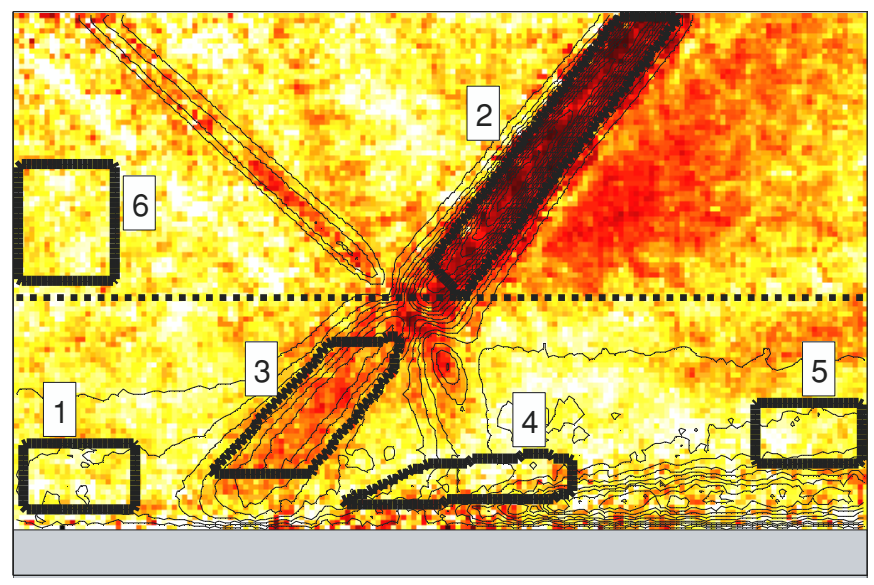

(a)

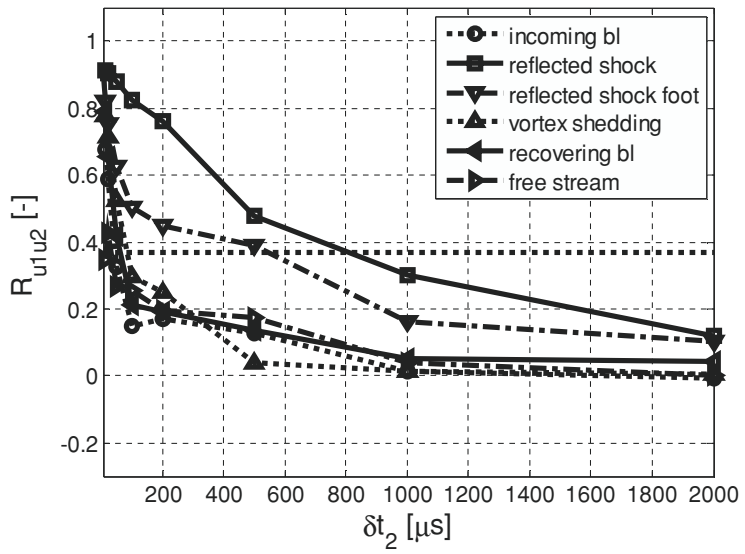

(b)

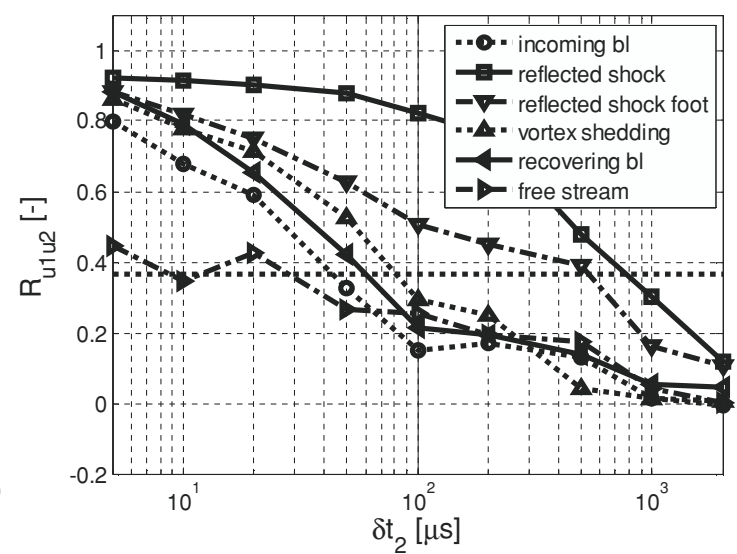

(c)

Figure 14. Local temporal auto-correlation coefficient for selected regions of the flow field: $(a)$ regions under consideration (1: incoming boundary layer, 2: reflected shock, 3: reflected shock foot, 4: vortex shedding, 5: recovering boundary layer, 6: free stream), Reynolds stress contour lines superimposed for reference; $(b)$ auto-correlation coefficient for each respective region; $(c)$ auto-correlation coefficient in semi-log representation.

this kind of interaction (see Dupont et al (2006) and Touber and Sandham (2008)).

\section{Conclusions}

A dual-PIV approach was used to study time-correlation phenomena in a shock-wave boundary layer interaction over a large range of time scales, including small values of the time delay that are not achievable by a single PIV system (maximum equivalent repetition rate $200 \mathrm{kHz}$ ). The physical alignment of the cameras has been verified by means of an evaluation of the homogeneous displacement field obtained from zerotime-delay measurements. The alignment was found to be of high quality (translational mismatch of the order of 1 pixel with an RMS of less than 1 pixel, rotational mismatch of five hundredth of a degree and a constant discrepancy in the magnification factor of the order of a tenth of a percent). The inter-camera statistical data compatibility is high and differences seem to be governed by statistical convergence uncertainties and PIV measurement uncertainties rather than camera misalignments. The dual-PIV measurements have been validated with respect to classical two component PIV measurements and with respect to semi-empirical relations for the friction coefficient and the friction velocity. It is concluded that the incoming boundary layer under consideration is a canonical turbulent boundary layer and that the flow conditions are repeatable. The dual-PIV dataset is of consistent quality and the spatial resolution is sufficient to capture the relevant flow physics. Measurement uncertainties in the mean velocity and the fluctuation components, either due to misalignments or due to PIV measurement errors, are limited to the near-wall region $(y / \delta<0.1)$. The Reynolds shear stress is accurately resolved down to $y / \delta<0.3$.

Based on the statistical and instantaneous dual-PIV velocity fields in combination with high-resolution interaction zoom data it can be concluded that the flow under consideration is a case of incipient separation. The temporal resolution of the dual-PIV measurements enabled the identification within the interaction of the downstream convection of vortical structures with a velocity of $U_{c}=290 \mathrm{~m} \mathrm{~s}^{-1}$.

Dual-PIV has been used to classify the time scales in the shock wave boundary layer interaction. This classification has shown that the time scales span almost three orders of magnitude. The smallest time scales are present in the 
incoming boundary layer. The obtained equivalent frequency of $25 \mathrm{kHz}$ seems to be underestimated with respect to the expected integral time scales. It is not known whether this is a physical or a measurement artefact. The frequencies associated with the $1 /$ e criterion of the autocorrelation function for the vortex shedding and the reflected shock, $12.5 \mathrm{kHz}$ and $1250 \mathrm{~Hz}$ respectively, seem to be in accordance with the literature. This confirms the low-frequency motion of the reflected shock.

The auto-correlation coefficient of the downstream boundary layer region follows the vortex-shedding trend for small time delays and converges towards the incoming boundary layer values for medium time delays. This could be indicative of the recovery of the boundary layer after the interaction. Finally, the results indicate that the shock foot moves in harmony with the low-frequency motion of shock in the outer flow.

The potential of dual-PIV to provide time-resolved measurements in high-speed flows has clearly been demonstrated. The achievability of small time delays combined with the high spatial resolution enabled the tracking of structures with convection speeds of several hundreds of meters per second. In addition, the large temporal dynamic range enabled us to simultaneously identify time scales of three orders of magnitude difference ( $1 \mathrm{kHz}$ up to $100 \mathrm{kHz}$ ) within a complete flow field containing over 20000 velocity vectors.

\section{Acknowledgments}

The current research was supported by the European Commission in the context of the 6th Framework Programme UFAST 'Unsteady effects in shock induced separation' (www.ufast.gda.pl). This work constitutes a cooperation between the Delft University of Technology (TU Delft) and the IUSTI laboratory (Université Aix-Marseille-CNRS, UMR CNRS No 6595) in Marseille. The experiments were performed at the TU Delft and the majority of the data processing took place at the IUSTI. The authors wish to gratefully acknowledge the input, ideas and support of both institutions.

\section{References}

Christensen K T and Adrian R J 2002a Measurement of instantaneous Eulerian acceleration fields by particle image accelerometry: method and accuracy Exp. Fluids 33 759-69

Christensen K T and Adrian R J 2002b The velocity and acceleration signatures of small-scale vortices in turbulent channel flow J. Turbul. 3023

Cousteix J 1989 Turbulence et Couche Limite (Toulouse: CEPADUE-Editions)

Doerffer P 2007 European research on unsteady effects of shock wave induced separation UFAST-project Proc. 8th Int. Symp. on Experimental and Computational Aerothermodynamics of Internal Flows (Lyon, July 2007) ISAIF8-0051

Dolling D S 2001 Fifty years of shock-wave/boundary-layer interaction research: what next? AIAA J. 39 1517-31

Dupont $\mathrm{P}$, Haddad C and Debiève J F 2006 Space and time organisation of a shock-induced separated boundary layer J. Fluid Mech. 559 255-77
Dupont P, Piponniau S, Sidorenko A and Debiève J F 2007 Investigation of an oblique shock reflection with separation by PIV measurements 45th AIAA Aerospace Sciences Meeting and Exhibit (Reno, USA, 8-11 January 2007)

Dussauge J P, Dupont P and Debiève J F 2006 Unsteadiness in shock wave turbulent boundary layer interactions with separations Aerosp. Sci. Technol. 10 85-91

Dussauge J P and Smits A J 1995 Characteristic scales for energetic eddies in turbulent supersonic boundary layers Proc. 10th Symp. on Turbulent Shear Flows (Pennsylvania State University

Elsinga G E, Van Oudheusden B W and Scarano F 2005 Evaluation of optical distortion effects in PIV Exp. Fluids $39246-56$

Fernholz H 1971 Ein halbempirisches Gesetz für die Wandreibung in kompressiblen turbulenten Grenzschichten bei isothermer und adiabater Wand Angew. Mech. ZAMM 51 146-7

Ganapathisubramani G, Clemens N T and Dolling D S 2007 High-speed PIV measurements to study the effect of upstream coherent structures on shock-induced turbulent separation 7 th Int. Sym. on Particle Image Velocimetry (Rome, Italy, 11-14 September 2007)

Garnier E and Sagaut P 2002 Large eddy simulation of shock/ boundary layer interaction AIAA J. 40 1935-44

Guibert P and Lemoyne L 2002 Dual particle image velocimetry for transient flow field measurements Exp. Fluids 33 355-67

Gurtin M E 1981 An Introduction to Continuum Mechanics (San Diego: Academic)

Humble R A, Scarano F and Van Oudheusden B W 2006 Experimental study of an unsteady impinging shockwave/turbulent boundary layer interaction using PIV 36th AIAA Fluid Dynamics Conf. and Exhibit (San Francisco, USA, 5-8 June 2006) AIAA-2006-3361

Kähler C J 2004 Investigation of the spatio-temporal flow structure in the buffer region of a turbulent boundary layer by means of multiplane stereo PIV Exp. Fluids 36 114-30

Klebanoff P S 1953 Characteristics of turbulence in a boundary layer with zero pressure gradient NACA Report 1247

Liu X and Katz J 2006 Instantaneous pressure and material acceleration measurements using a four-exposure PIV system Exp. Fluids $\mathbf{4 1} 227-40$

Perret L, Braud P, Fourmet C, David L and Delville J 2006 3-Component acceleration field measurement by dual-time stereoscopic particle image velocimetry Exp. Fluids 40 813-24

Pirozzoli S and Grasso F 2006 Direct numerical simulation of impinging shock wave/turbulent boundary layer interaction at $M=2.25$ Phys. Fluids 18065113

Scarano F and Riethmuller M L 1999 Iterative multigrid approach in PIV image processing with discrete window offset Exp. Fluids 26 513-23

Smits A J 1997 Compressible turbulent boundary layers AGARD FDP Special Course on Turbulence in Compressible flows (Von Kármán Institute for Fluid Dynamics, Belgium, 2-6 June 1997) AGARD-R-819

Smits A J and Dussauge J P 2006 Turbulent Shear Layers in Supersonic Flow 2nd edn (New York: Springer)

Souverein L J, Van Oudheusden B W and Scarano F 2007a Particle image velocimetry based loads determination in supersonic flows 45th AIAA Aerospace Sciences Meeting and Exhibit (Reno, USA, 8-11 January 2007) AIAA-2007-0050

Souverein L J, Van Oudheusden B W and Scarano F 2007b Determination of time scales in a shock wave turbulent boundary layer interaction by means of dual PIV 7th Int. Symp. on Particle Image Velocimetry (Rome, Italy, 11-14 September 2007)

Souverein L J, Van Oudheusden B W, Scarano F and Dupont P 2008 Unsteadiness characterization in a shock wave turbulent boundary layer interaction through dual PIV 38th AIAA Fluid 
Dynamics Conf. (Seattle, USA, 23-26 June 2008) AIAA-2008-4169

Touber E and Sandham N D 2008 Oblique shock impinging on a turbulent boundary layer: low-frequency mechanisms $38 \mathrm{th}$ AIAA Fluid Dynamics Conf. (Seattle, USA, 23-26 June 2008) AIAA-2008-4170

Van Oudheusden B W, Scarano F, Roosenboom E W M, Casimiri E W F and Souverein L J 2007 Evaluation of integral forces and pressure fields from planar velocimetry data for incompressible and compressible flows Exp. Fluids 43 153-62

Van Oudheusden B W and Souverein L J 2007 Evaluation of the pressure field from PIV in a shock wave boundary layer interaction 7th Int. Symp. on Particle Image Velocimetry (Rome, Italy, 11-14 September 2007)

White F M 1991 Viscous Fluid Flow 2nd edn (New York: McGraw-Hill) 